\title{
Gastric Microbiota in Helicobacter pylori-Negative and -Positive Gastritis Among High Incidence of Gastric Cancer Area
}

\author{
Boldbaatar Gantuya ${ }^{1,2}$, Hashem B. El-Serag ${ }^{3}$, Takashi Matsumoto ${ }^{1}{ }^{\mathbb{D}}$, Nadim J. Ajami ${ }^{4}$, \\ Khasag Oyuntsetseg ${ }^{2}$, Dashdorj Azzaya ${ }^{1,2}$, Tomohisa Uchida ${ }^{5}$ and Yoshio Yamaoka $1,3,6, *$ (D) \\ 1 Department of Environmental and Preventive Medicine, Oita University Faculty of Medicine, 1-1 Idaigaoka, \\ Hasama-machi, Yufu-City, Oita 879-5593, Japan; medication_bg@yahoo.com (B.G.); \\ tmatsumoto9@oita-u.ac.jp (T.M.); azzaya2000@gmail.com (D.A.) \\ 2 Department of Internal Medicine, Gastroenterology Unit, Mongolian National University of Medical \\ Sciences, Zorig Street, Ulaanbaatar-14210, Mongolia; oyuntsetseg.kh@mnums.edu.mn \\ 3 Department of Medicine, Gastroenterology and Hepatology Section, Baylor College of Medicine, \\ 7200 Cambridge Street, Houston, TX 77030, USA; hasheme@bcm.edu \\ 4 Alkek Center for Metagenomics and Microbiome Research, Department of Molecular Virology and \\ Microbiology, Baylor College of Medicine, Houston, TX 77030, USA; nadimajami@gmail.com \\ 5 Department of Molecular Pathology, Oita University Faculty of Medicine, 1-1 Idaigaoka, Hasama-machi, \\ Yufu-City, Oita 879-5593, Japan; tomohisa@oita-u.ac.jp \\ 6 Global Oita Medical Advanced Research Center for Health, 1-1 Idaigaoka, Hasama-machi, Yufu-City, \\ Oita 879-5593, Japan \\ * Correspondence: yyamaoka@oita-u.ac.jp; Tel.: +81-97-586-5740; Fax: +81-97-586-5749
}

Received: 9 March 2019; Accepted: 4 April 2019; Published: 10 April 2019

\begin{abstract}
Helicobacter pylori (H. pylori) related chronic gastritis is a well-known major etiological factor for gastric cancer development. However, $H$. pylori-negative gastritis $(\mathrm{HpN})$ is not well described. We aimed to examine gastric mucosal microbiota in $\mathrm{HpN}$ compared to $\mathrm{H}$. pylori-positive gastritis $(\mathrm{HpP})$ and $\mathrm{H}$. pylori-negative non-gastritis group (control). Here, we studied 11 subjects with $\mathrm{HpN}$, 40 with $\mathrm{HpP}$ and 24 controls. We performed endoscopy with six gastric biopsies. Comparison groups were defined based on strict histological criteria for the disease and H. pylori diagnosis. We used 16S rRNA gene amplicon sequencing to profile the gastric microbiota according to comparison groups. These results demonstrate that the HpP group had significantly lower bacterial richness by the operational taxonomic unit (OTU) counts, and Shannon and Simpson indices as compared to $\mathrm{HpN}$ or controls. The linear discriminant analysis effect size analysis showed the enrichment of Firmicutes, Fusobacteria, Bacteroidetes and Actinobacteria at phylum level in the HpN group. In the age-adjusted multivariate analysis, Streptococcus sp. and Haemophilus parainfluenzae were at a significantly increased risk for $\mathrm{HpN}$ (odds ratio 18.9 and 12.3, respectively) based on abundance. Treponema sp. was uniquely found in $\mathrm{HpN}$ based on occurrence. In this paper, we conclude that Streptococcus sp., Haemophilus parainfluenzae and Treponema sp. are candidate pathogenic bacterial species for HpN. These results if confirmed may have important clinical implications.
\end{abstract}

Keywords: Helicobacter pylori; non-Helicobacter pylori; gastritis; microbiota; $16 \mathrm{~S}$ rRNA

\section{Introduction}

Most of the gastric cancer incidence is arising from East-Asian countries. Among them the leading countries are Korea, followed by Mongolian and Japan. By the mortality rate the worst country is Mongolia. It is reported that $97 \%$ of overall gastric cancer belonged to the sporadic type [1]. Gastric 
cancer is the multifactorial disease however the Helicobacter pylori (H. pylori) infection is considered a major etiological factor [2]. The development of next generation sequencing technologies allow for in depth studies of the gastric microbiota composition. A recent review paper for gastric cancer microbiota highlighted Escherichia Shigella and Burkholderia within the Proteobacteria phylum; Lactobacillus, Lachnospiraceae, Streptococcus, and Veillonella within the Firmicutes phylum; and Prevotella within the Bacteroidetes phylum. Although great advances have been made in understanding the complex interplay between the gastric microbiota and H. pylori in the development of gastric inflammation and cancer, detailed studies are still needed in well-defined human populations to compare differences in the microbiota of $H$. pylori-infected persons with and without neoplastic lesions [3].

For the gastric cancer pathogenesis, H. pylori plays the main role for developing gastric cancer due to atrophic gastritis, which mainly develop an intestinal type gastric cancer and non-atrophic gastritis, which mainly develop a diffuse type gastric cancer [1,4]. Gastritis is a histopathological entity that is characterized by neutrophilic infiltration. Previous studies reported that $H$. pylori-negative gastritis is a common histopathological and clinical condition that is independent from H. pylori gastritis in US populations [5]. A recent study showed a 24-fold increase in neutrophil counts in the gastric cancer tissue, and nine-fold increase in gastric intestinal metaplasia tissue compared to the normal gastric tissue. H. pylori stimulates the neutrophil accumulation in epithelial cells with the production of inflammatory mediators, reactive oxygen and nitrogen species, which contribute to the disruption of gastric epithelial function [6] and DNA damage [7]. Neutrophil infiltration is also associated with the E-cadherin downregulation, cell proliferation and gastric carcinogenesis [8]. While it is well known that gastritis is induced by the H. pylori infection, [9] the role of other bacterial infections of the stomach is not well studied.

The composition and diversity of microbial communities in the stomach is greatly influenced by gastric acidity [10]. A well-established factor influencing gastric acidity is the H. pylori infection, which triggers the development of chronic active gastritis that may subsequently progress to chronic atrophic gastritis. Previous studies using the $16 \mathrm{~S}$ rRNA gene sequencing, reported that the gastric microbiota diversity in gastric tissue samples was dramatically reduced in $H$. pylori-positive cases compared to negative cases [11]. H. pylori comprised most (the average $72 \%$ ) of sequencing reads among patients with positive conventional $H$. pylori tests (i.e., culture, rapid urease test, serology and histology) [12]. However, the gastritis status was not examined in the study. Another study reported a high abundance of Streptococcus sp. in the H. pylori-negative antral gastritis. However, the definition of $H$. pylori negative cases in that study was based on the rapid urease test and $16 \mathrm{~S}$ rRNA gene sequencing, and the gastritis diagnosis was based on endoscopic findings and presence or absence of gastric atrophy but not neutrophils. Furthermore, the number of subjects examined was small (five cases and five controls) [13]. Lastly, the gastric microbiome and acidity are also modified by antibiotics, proton pump inhibitors (PPIs) and gastric surgery [14,15]. Therefore, there have been no studies of the gastric microbiome in $H$. pylori-negative gastritis that considers a larger number of subjects with careful definitions of the H. pylori infection, gastritis and previous medical history.

To address this important gap in the literature, we examined differences in the gastric microbiome for $H$. pylori-negative gastritis $(\mathrm{HpN})$ compared to the $H$. pylori-positive gastritis $(\mathrm{HpP})$ and H. pylori-negative non-gastritis group (control) in the Mongolian population employing rigorous definitions for cases and controls.

\section{Results}

\subsection{Study Population}

Samples were collected and sequenced for microbiome profiling from 220 subjects. Of these, 145 cases were excluded due to our criteria for defining study groups. The analysis presented here pertains to samples from a total of 75 subjects $(11 \mathrm{HpN}, 40 \mathrm{HpP}$, and 24 controls). The HpN group was defined by the positive histological diagnosis for gastritis and negative by all $H$. pylori tests including $16 \mathrm{~S}$ rRNA 
gene amplicon sequencing. The $\mathrm{HpP}$ group was positive by the histological diagnosis for gastritis and positive by all $\mathrm{H}$. pylori tests including $16 \mathrm{~S}$ rRNA gene amplicon sequencing. The control group was based on the histological diagnosis that neutrophil infiltration was not seen in the gastric mucosa, Updated Sydney system score 0 (none of infiltration) for gastritis and negative by all $H$. pylori tests including 16S rRNA gene amplicon sequencing. The flow chart of the sample selection is shown in Supplementary Materials Figure S1. The overall mean age was 46.8 years (standard deviation 15.6), and $72 \%$ were females. There were no significant age or gender differences among the three groups (Table S1).

\subsection{Detection of Operational Taxonomic Units (OTUs)}

We identified a total 2,550 OTUs of which 125 OTUs were filtered based on OTU low abundance (minimum total count $<10$ or minimum relative abundance $0.05 \%$ of OTUs as a default option of the CLC genomic workbench 8.5.4 version for microbiome analysis). The overall count reads per sample was 6,181,354 OTU reads (minimum 2,053 and maximum 227,528), and the dataset was rarefied to 2,053 OTU reads per sample (Figure S2). The minimum total count per OTU was 41 (Fusobacterium OTU ID: 828676) and maximum was 4,513,446 OTU reads (H. pylori OTU ID: 10952).

The rarefaction analysis is shown for $\mathrm{HpN}$ (Figure S3A), HpP (Figure S3B) and control (Figure S3C) groups, respectively. Most $\mathrm{HpN}$ group cases had higher bacterial diversity (richness) (Overall OTU relative abundance scaled up to $100 \%$ ) but possessed lower read numbers (Scaled by raw count numbers up to 200,000). HpP group cases had a variety of richness and had generally higher read numbers per sample. The cases of control group had a variety of richness and had relatively lower read numbers per sample.

Recovered taxonomies (Figure S4A) and their relative abundance are shown at genus level per sample (Figure $\mathrm{S4B}$ ). Cases with $\mathrm{HpN}$ and control groups had a higher bacterial diversity but were observed to have a lower read count compared to the HpP group. The HpP group had overall less diversity due to the dominance of Helicobacter genus as observed by an increased read count mapping to the Helicobacter genus. One $\mathrm{HpN}$ case showed a striking dominance by Treponema. The sequenced data will be deposited on the Genbank as Accession Number (in proceeding).

\subsection{Microbiome Diversity}

Microbial community richness and evenness as represented by Shannon (Figure 1A), richness (Figure 1B) and Simpson's diversity indices (Figure 1C) showed statistically significant differences between the $\mathrm{HpN}$ vs. $\mathrm{HpP}$, and for $\mathrm{HpP}$ vs. control, but not for the $\mathrm{HpN}$ vs. control group comparison. The beta-diversity analysis using Bray-Curtis distance metrics revealed differences in the microbial structure between the $\mathrm{HpP}$ and $\mathrm{HpN}$ groups (R2 0.5, $p<0.0003$ ), but no significant differences between the $\mathrm{HpN}$ and control groups ( $\mathrm{R} 20.05, p=0.2$ ). Similarly, the Jaccard distance metric was highly significant $(\mathrm{R} 20.47, p<0.0003)$ for the $\mathrm{HpN}$ vs. HpP group comparison but not for the $\mathrm{HpN}$ vs. control group (R2 0.04, $p=0.1$ ). The Discriminant Analysis of Principal Components (DAPC) analysis used to identify the group level clusters showed a clear separation among the three groups. The HpP group had a more distinct composition compared to the $\mathrm{HpN}$ or control groups, which were closer to each other in composition (Figure 1D). These differences were driven by the absence or presence of taxonomies in which 120 were determined in each group to be part of a core microbiome, control and HpP groups shared 3 taxa (Enterococcus sp. OTU ID: 766768, Lactobacillus sp. OTU ID: 324926, Lactobacillus sp. OTU ID: 851733), HpN and HpP shared one OTU (Fusobacterium sp. OTU ID: 828676) and one OTU (Treponema sp. OTU ID: 2707164) was unique in the HpN group (Figure 1E). Then, we described the differences of each group based on abundance. 
A

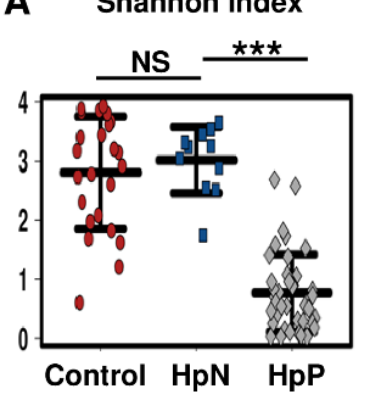

D

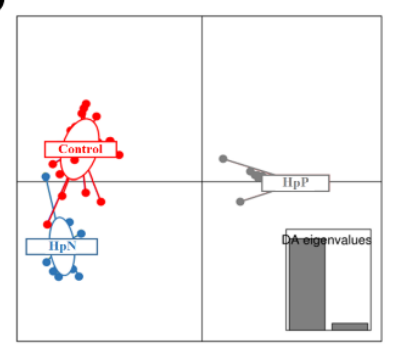

B

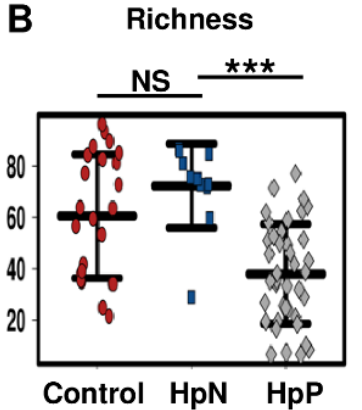

E

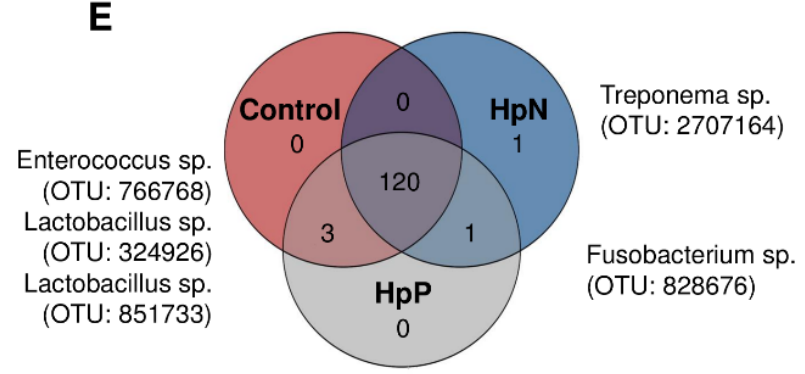

Figure 1. Alpha diversity and unique taxonomy by comparison groups. Shannon index (A); bacterial richness (B); Simpson's index (C); (D) discriminant analysis of principal components (DAPC); (E) Venn diagram for overall operational taxonomic units (OTU) taxonomy are shown according to HpN, $\mathrm{HpP}$ and control groups. Venn diagram based on the absence or presence of taxonomies showed 120 species were core, four species were pan and one species were determined as unique taxonomy among comparison groups. NS: not significant, ${ }^{* * *} p<0.001$.

The alpha diversity analysis showed that among the HpP group, the H. pylori relative abundance was around $90 \%$, whereas bacterial taxa were more diverse among $\mathrm{HpN}$ and control groups. The overall relative abundance is shown for phylum level (Figure 2A) and for genus level (Figure 2B). Differences in averaged bacterial abundance were determined by analysis of variance (ANOVA). Three Streptococcus OTUs had significantly higher abundance in $\mathrm{HpN}$ than the control group (Figure S5A). For the HpP vs. HpN comparison, H. pylori was higher in $\mathrm{HpP}$ whereas most of the remaining OTU taxonomies were significantly higher in the HpN group. Top $10 \mathrm{OTU}$ taxonomies are shown for HpP and $\mathrm{HpN}$ comparison (Figure S5B).

Biomarker discovery using the linear discriminant analysis effect size (LEfSe) algorithm showed that Proteobacteria was enriched in the HpP group; and Firmicutes, Bacteroidetes, Fusobacteria and Actinobacteria were enriched in the $\mathrm{HpN}$ group at phylum level (Figure 2C). Helicobacter was the only distinguishing taxon in the $\mathrm{HpP}$ group, whereas 14 distinct taxa distinguished $\mathrm{HpN}$ from the $\mathrm{HpP}$ and control groups. At the genus level, nine taxa distinguished the control group from the rest (Figure 2D). Using the Wilcoxon test, we found 10 species as potential biomarkers for the HpN compared with control as well as the HpP group that Streptococcus sp. (OTU ID: 1010458, ID: 1078207, ID: 526131, ID: 967427, ID: 989579, ID: 525966), Haemophilus parainfluenzae (OTU ID: 4320756), Fusobacterium (OTU ID: 938948), Veillonella (OTU ID: 511378) and Prevotella Pallens (OTU ID: 705241) (Table S2).

The network analysis showed bacterial interactions among all three-comparison groups (Figure S6A), the $\mathrm{HpN}$ and control (Figure S6B), and the HpN and HpP groups (Figure S6C). Similar to the overall relative abundance, $H$. pylori did not co-occur with other taxa and dominated in the $\mathrm{HpP}$. High microbial co-occurrence was observed in the $\mathrm{HpN}$ and the control group suggestive of a richer community. 
A

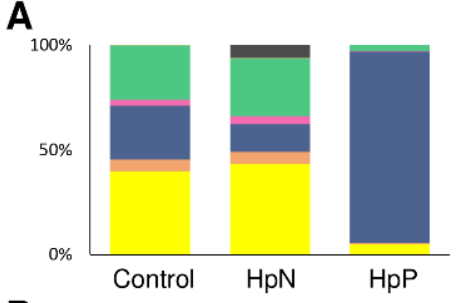

B

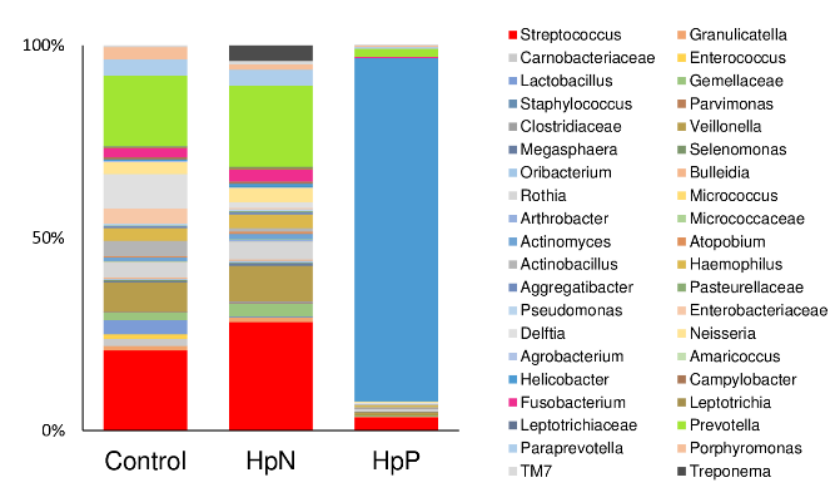

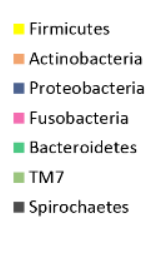

(1)

C

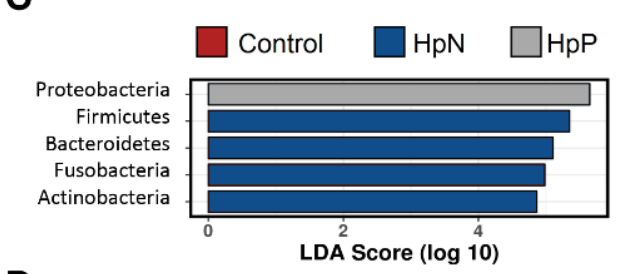

D

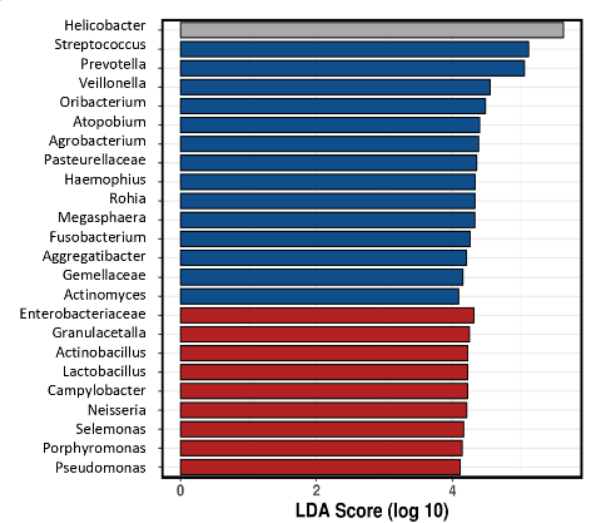

Figure 2. Relative abundance and linear discriminant analysis effect size (LEfSe) by comparison for groups. Microbial relative abundance percentages of the gastric microbiome shown for phylum (A) level, genus level (B); LEfSe test at phylum level (C); at genus level (D) are shown according to the $\mathrm{HpN}, \mathrm{HpP}$ and control groups.

\subsection{Univariate, Receiver Operating Characteristics (ROC) Curve and Multivariate Analysis}

From the biomarker discovery analysis (Table S2) between $\mathrm{HpN}$ vs. $\mathrm{HpP}$ and $\mathrm{HpN}$ vs. control, we selected the 10 candidate species (for HpN (described above)), which were consistent in both comparisons between $\mathrm{HpN}$ vs. $\mathrm{HpP}$, and $\mathrm{HpN}$ vs. control group. The average abundance and statistical significance based on the $t$-test among the $\mathrm{HpN}, \mathrm{HpP}$ and control groups is shown for each candidate (Figure 3).
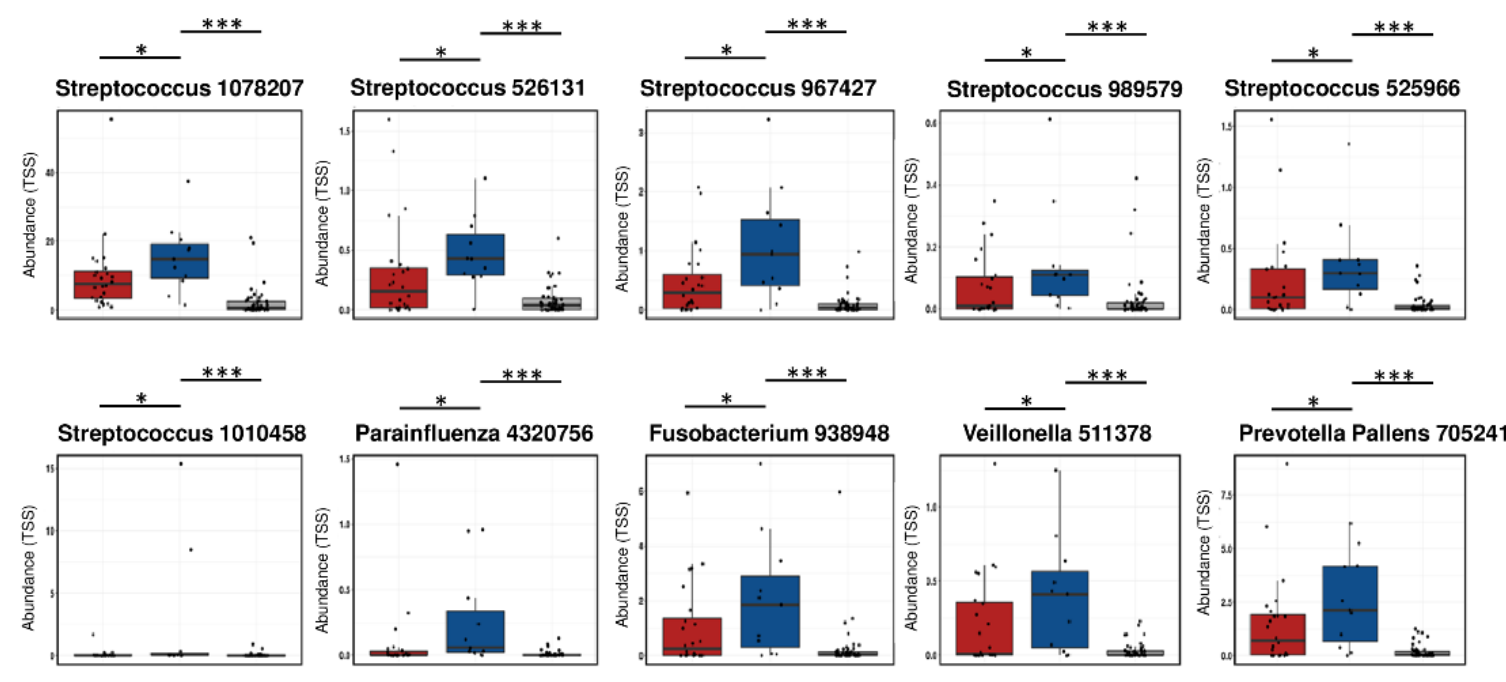

Figure 3. Univariate analysis for selected species according to comparison groups. Each selected species of abundance was shown by the $\mathrm{HpN}, \mathrm{HpP}$ and control groups. ${ }^{*} p<0.05,{ }^{* * *} p<0.001$.

All candidates were significantly higher in $\mathrm{HpN}$ than the control group followed by the HpP group. Additionally, Treponema (OTU ID: 2707164) was significantly higher in the HpN group compared 
with the HpP group by ANOVA (Figure 4A) but not by the LEfSe. This result was driven by the high abundance of Treponema observed in one $\mathrm{HpN}$ case. The arithmetic mean \pm standard deviation of the relative abundance (raw count number reads) were $5.9 \pm 19.5(1736 \pm 5758)$ for $\mathrm{HpN}, 0 \pm 0$ $(0.02 \pm 0.1)$ for $\mathrm{HpP}$, and $0 \pm 0(0 \pm 0)$ for controls $(p<0.036)$. Similar to the case of $H$. pylori in the HpP group, Treponema (OTU ID: 2707164) strongly dominated over other taxa in that sample (Figure 4B). Inflammatory cell accumulation with hemorrhagic change was observed in the gastric histopathology of such Treponema positive by the $16 \mathrm{~S}$ rRNA patient (Figure 4C).
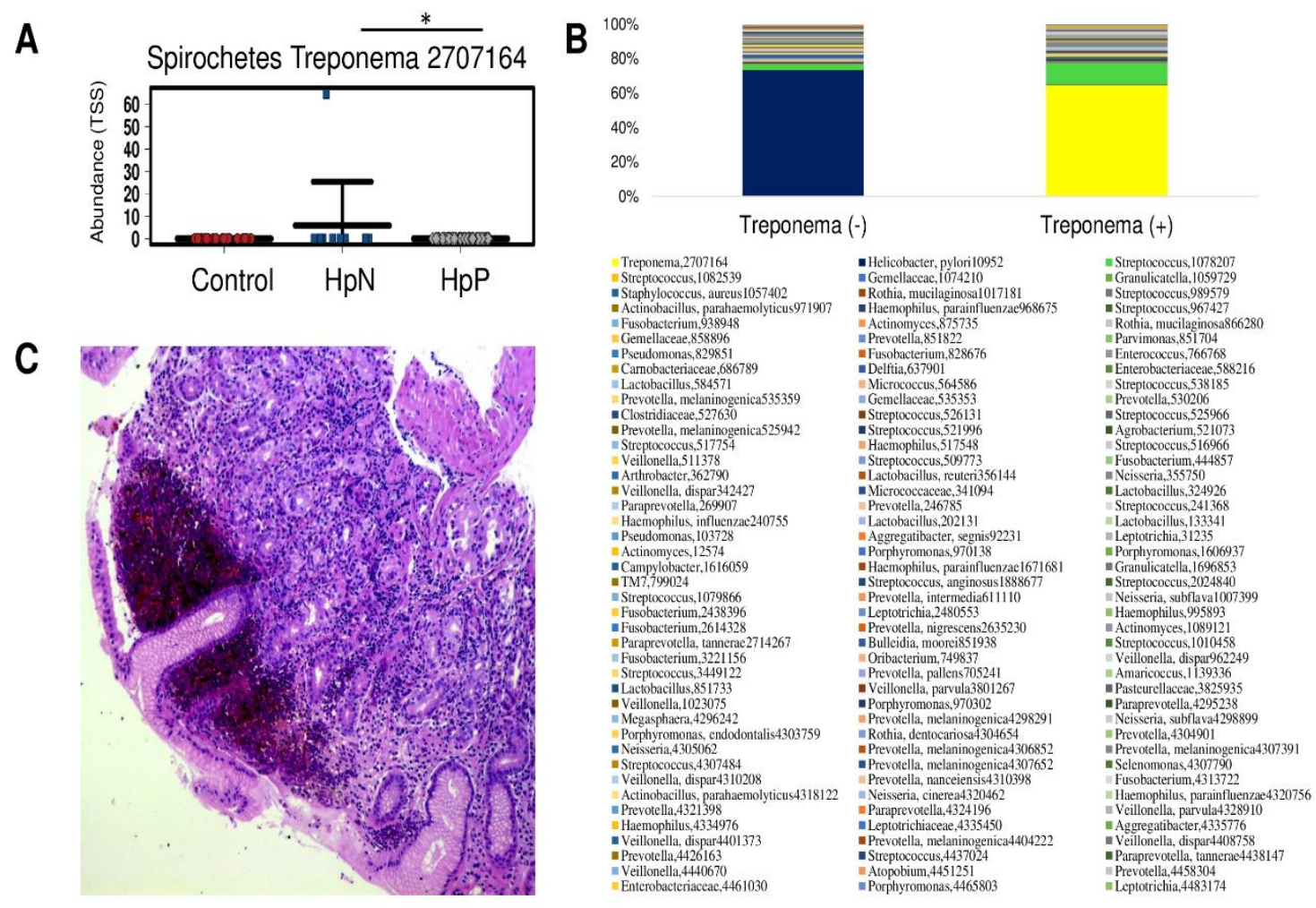

Figure 4. The rare case of syphilitic gastritis and its relative abundance. The mean abundance value of Treponema (OUT ID 2707164) at the species level was high in $\mathrm{HpN}$ compared with the HpP and control group (A); its relative abundance is shown for Treponema negative and Treponema positive cases (B) with its gastric histopathological image (C). Inflammatory cell accumulation with hemorrhagic change was observed by hematoxylin and eosin staining in Treponema positive patient by $16 \mathrm{~S}$ rRNA. ${ }^{*} p<0.05$.

We selected the 10 candidate species that were significantly enriched in $\mathrm{HpN}$ comparing with each of the $\mathrm{HpP}$ and control group using Wilcoxon tests. In addition, H. pylori were included. Area under curve (AUC) was $<0.7$ for Streptococcus (OTU ID: 989579), Prevotella (OTU ID: 705241) and Treponema (OTU ID: 2707164) which were excluded. The remaining candidate species were included in the multivariate analysis. The selected candidates are shown in the receiver operating characteristics (ROC) curve (Figure S7) and the AUC, best cut off values in Table 1.

Finally, Streptococcus (OTU ID: 525966) and Haemophilus parainfluenza OTU ID: 4320756) remained as the strongest significant candidates for the $\mathrm{HpN}$ group in the age adjusted backward multivariate logistic regression analysis (Table 2). 
Table 1. The best cut off points for selected microbial candidates of $H$. pylori-negative gastritis.

\begin{tabular}{lccccccc}
\hline \multicolumn{1}{c}{ OTU } & Area & $\begin{array}{c}\text { Lower } \\
\text { Bound }\end{array}$ & $\begin{array}{c}\text { Upper } \\
\text { Bound }\end{array}$ & $\begin{array}{c}\text { Cut off } \\
\text { Value }\end{array}$ & $\begin{array}{c}\text { Sensi- } \\
\text { Tivity }\end{array}$ & $\begin{array}{c}\text { Speci- } \\
\text { Ficity }\end{array}$ & $p$ Value \\
\hline Streptococcus 1078207 & 0.74 & 0.58 & 0.9 & 3099 & 0.64 & 0.28 & 0.011 \\
Streptococcus 989579 & 0.68 & 0.53 & 0.82 & & & - & NS \\
Streptococcus 967427 & 0.77 & 0.6 & 0.93 & 138 & 0.73 & 0.27 & 0.005 \\
Streptococcus 526131 & 0.74 & 0.59 & 0.88 & 90.5 & 0.73 & 0.27 & 0.013 \\
Streptococcus 1010458 & 0.73 & 0.57 & 0.89 & 4.5 & 0.64 & 0.34 & 0.015 \\
Streptococcus 525966 & 0.83 & 0.72 & 0.94 & 64.5 & 0.82 & 0.19 & 0.001 \\
Fusobacterium 938948 & 0.73 & 0.55 & 0.91 & 177.5 & 0.73 & 0.31 & 0.014 \\
Veillonella 511378 & 0.78 & 0.63 & 0.92 & 46 & 0.73 & 0.27 & 0.003 \\
Prevotella pallens 705241 & 0.68 & 0.49 & 0.88 & & & - & NS \\
Haemophilus & 0.71 & 0.54 & 0.89 & 6.5 & 0.73 & 0.27 & 0.025 \\
parainfluenzae 4320756 & & & & & & \\
Helicobacter pylori 10952 & 0.75 & 0.65 & 0.86 & 164.5 & 0.36 & 0.7 & 0.008 \\
\hline
\end{tabular}

NS: $p$ value is not statistically significant $(p>0.05)$.

Table 2. Age adjusted multivariate logistic regression analysis of microbial biomarkers for H. pylorinegative gastritis.

\begin{tabular}{lcccc}
\hline \multicolumn{1}{c}{ OTU } & $\boldsymbol{p}$ Value & Odds Ratio & Lower 95\% C.I. & Upper 95\% C.I. \\
\hline Streptococcus 525966 & 0.009 & 18.9 & 2.1 & 172.8 \\
Haemophilus parainfluenzae 4320756 & 0.025 & 12.3 & 1.4 & 109.6 \\
Fusobacterium 938948 & NS & 7.5 & 0.6 & 86.7 \\
Veillonella 511378 & NS & 5.9 & 0.7 & 51.4 \\
Helicobacter pylori 10952 & 0.028 & 0.1 & 0.0 & 0.8 \\
\hline
\end{tabular}

NS: $p$ value is not statistically significant $(p>0.05)$.

\section{Discussion}

H. pylori-negative gastritis $(\mathrm{HpN})$ is relatively common $(1.5-21 \%$ of all gastritis) and while it is different and independent from the $H$. pylori infection $[5,16]$ it shares similar at least short-term clinical implications. The etiology of $\mathrm{HpN}$ are is not well described [16] and it is possible that other bacterial infections are responsible. In this study, we have described the composition of microbiota in gastric mucosal samples of individuals with $\mathrm{HpN}$ compared to two control groups $(H$. pylori-positive gastritis $(\mathrm{HpP})$ and H. pylori-negative non-gastritis (control)). The three groups were strictly defined based on multiple diagnostic tests (for the H. pylori status) and updated Sydney system (for the gastritis status). Our findings show significant bacterial differences among the three groups that may contribute to the pathogenesis of gastritis, and highlight Streptococcus (OTU ID: 525966), Haemophilus parainfluenza (OTU ID: 4320756) and Treponema (OTU ID: 2707164) as possible unique markers of HpN gastritis. These findings if confirmed may have important clinical implications.

The Kyoto global consensus conference summary acknowledges the specific bacterial etiology-based classification of gastritis not just the $H$. pylori infection but also other bacteria such as H. heilmannii, Enterococcus, Mycobacteria and Syphilis [17]. Several studies examined gastric mucosal microbiota in H. pylori-negative and H. pylori-positive patients with gastritis, atrophy and gastric cancer, however, none of these studies considered strict definitions of the $H$. pylori negative status or a histopathological definition of gastritis based on neutrophil infiltration score [11,18]. Strains possess strong stimulatory capacity for neutrophil activation which may play the role in the pathogenesis of gastritis [19]. A recent culture-based study of gastric mucosal tissue showed that Streptococcus as well as Neisseria were markedly higher in the gastritis group than normal controls [20]. Streptococcal infection was also implicated in the pathogenesis of the rare but fatal phlegmonous gastritis [21]. Furthermore, several studies highlighted that the Streptococcal infection was significantly associated with gastric cancer [3]. 
The second bacterial candidate for $\mathrm{HpN}$ group in our study was Haemophilus parainfluenzae, which is an opportunistic pathogen responsible for several infections [22], including the respiratory tract infections [23], endocarditis [24], bacteremia and sepsis [25]. Previous studies reported the detection of lower reads of Haemophilus parainfluenzae in the stomach; [26] however it was unclear whether or not it was associated with gastritis. One previous study highlighted that Haemophilus parainfluenzae was one of the predominant species among gastric cancer patients based on 16S rRNA amplicon sequencing approach [27]. Importantly, a previous clinical experimental study demonstrated that the Haemophilus parainfluenzae bacterial isolation from achlorhydric stomach patients had to increase nitrite accumulation which is a precursor of the carcinogenic N-nitroso compounds that formed in the gastrointestinal tract by a combination of chemical and enzymic reactions, and that the longer it persists, the greater the tendency for the carcinogens to be formed [28].

Lastly, one HpN subject in our study was infected with Treponema. Syphilitic gastritis is one of the rare manifestations of syphilis. Our study showed that similar to H. pylori infection, Treponema dominated other microbiota with a relative abundance of $65 \%$ [29].

The gastric microbiome is influenced by other factors that affect gastric acidity such as gastric surgery and PPIs use, which altered gastric microbiota with a shift towards a less healthy microbiome $[15,30]$. Therefore, a strength of our study is that the results were not confounded by these clinical variables because we excluded patients with antibiotic or acid inhibitors use. The study had few limitations including the limited sample size especially for those with $\mathrm{HpN}$ due to the stringent selection criteria used. However, we elected to pursue clearly defined groups and plan to pursue these promising preliminary findings in a larger study. It is also possible that the findings in a Mongolian population are not be generalizable to other populations due to environmental (e.g., diet) or host genetic differences. Lastly, the cross-sectional design precludes causal inferences.

Based on our finding and published literature we hypothesize that pathogenic candidates which are Streptococcus and Neisseria may play a role for gastric cancer development other than the H. pylori infection.

\section{Materials and Methods}

\subsection{Study Population and Sampling}

We conducted a cross-sectional study of subjects prospectively recruited during November 2014 to August 2016 from the Uvs Province (Western part of Mongolia), Khuvsgul Province (Northern), Umnugovi Province (Southern) and Khentii Province (Eastern Mongolia). Study subjects were recruited from volunteers with dyspepsia symptoms through community-based advertisements. We included only those age $\geq 18$ years old and with no history of antibiotic or acid inhibitor use within the prior six months. We excluded subjects with a history of gastrectomy, endoscopic mucosal dissection or H. pylori eradication therapy. The gastric biopsy protocol consisted of six specimens: Three from the gastric antrum for microbial examination (one for $H$. pylori rapid urease test, one for H. pylori culture and one for microbiome study) and three for histopathological examination (one from the angulus corpus-antrum junction, one from the greater curvature of the corpus and one from the greater curvature of the antrum). The sampling protocol was based on the American Society for Gastrointestinal Endoscopy guideline [31]. Written informed consent was obtained from all participants, and the ethical permission was approved by the Mongolian Ministry of Health (accepted number N3, 2015) Mongolian National University of Medical Sciences (N13-02/1A, 2013), and Oita University Faculty of Medicine (Yufu, Japan) (P-12-10, 2013).

\subsection{Histological Examination and Gastritis Definition}

The gastric biopsy specimens for histological examination were collected in separate tubes containing 10\% formaldehyde and kept at room temperature. Serial sections were stained with the hematoxylin eosin and with May-Giemsa stain. An experienced pathologist (T.U.) examined the 
stained slides and recorded the findings. The degree of neutrophil infiltration was evaluated in antrum, corpus and incisura angulus. The scores were 0 "normal", 1 "mild", 2 "moderate", and 3 "marked" based on the updated Sydney system [32]. A score $\geq 1$ was considered as positive for gastritis in any gastric biopsy site.

\subsection{Determination of $H$. pylori Infection}

Biopsy specimen for the $H$. pylori culture was immediately placed in a dram vial containing $1 \mathrm{~mL}$ of cysteine transport medium with $20 \%$ glycerol. [33] The collected samples were placed in $-20{ }^{\circ} \mathrm{C}$ temporarily then kept at $-80^{\circ} \mathrm{C}$. Collected samples were transported from Mongolia to Japan with dry ice. H. pylori was initially evaluated using five conventional diagnostic tests (culture, histology, immunohistochemistry, serology and rapid urease test). For the H. pylori culture, the antral biopsy specimen was homogenized in normal saline solution and placed in a commercially available selective plate (Nissui Pharmaceutical Co. Ltd., Tokyo, Japan). The plates were incubated for up to 10 days at $37^{\circ} \mathrm{C}$ under microaerophilic conditions ( $10 \% \mathrm{O} 2,5 \% \mathrm{CO} 2$, and $\left.85 \% \mathrm{~N} 2\right)$. H. pylori was identified based on colony morphology, Gram staining, and positive reactions for urease tests.

The H. pylori load was classified into four grades: 0 "normal", 1 "mild", 2 "moderate", and 3 "marked" based on the updated Sydney system [32]. A load $\geq$ grade 1 was considered positive for H. pylori. Immunohistochemistry commercially available anti-H. pylori antibody (Dako, Glostrup, Denmark) was used to confirm the H. pylori infection [34]. Serology of the H. pylori infection was evaluated using a commercially available anti-H. pylori IgG antibody ELISA kit (Eiken Co., Ltd., Tokyo, Japan). The $H$. pylori status by $16 \mathrm{~S}$ rRNA gene sequencing using a relative abundance cut off value of $2 \%$ for positive results was based on a previous study [35].

\subsection{DNA Extraction and Sequencing}

Biopsy specimen for microbiome analysis was collected in $0.5 \mathrm{~mL}$ Allprotect Tissue Reagent buffer (QIAGEN, Hilden, Germany). The collected samples were placed in $-20{ }^{\circ} \mathrm{C}$ then transported from Mongolia to Japan on dry ice. We performed the DNA extraction and purification using the QIAGEN kit according to the manufacturer's instruction. Amplicon libraries for pair-end $(2 \times 300 \mathrm{bp})$ sequencing on the Illumina MiSeq platform (Illumina Inc., San Diego CA, USA) were constructed using universal primers targeted across the V3 and V4 hypervariable regions of the 16S rRNA gene. The 16S rRNA gene was amplified using primers 341F $5^{\prime}$-CCTACGGGNGGCWGCAG-3' and 805R $5^{\prime}$-GACTACNVGGGTATCTAATCC- ${ }^{\prime}$ which include overhang adapter sequences at the $5^{\prime}$ end to add multiplexing indexes. Libraries were cleaned using Agencourt Ampure XP beads according to the manufacturer's instruction and sequenced on an Illumina MiSeq platform. The bioanalyzer MCE-202 MultiNA system (Shimadzu, Kyoto, Japan) and QuantFluor dsDNA system (Promega Corporation, Madison, WI, USA) were used to examine the quality of sample. All good quality DNA samples were normalized as the same amount.

\subsection{Sequence Curation and Analysis}

Trimming and quality filtering of the 16S rRNA gene sequence data was performed with the CLC genomic workbench 8.5.4 version (QIAGEN). After sequencing for further analysis our criteria for the minimum OTU reads was less than 10 or the minimum relative abundance is less than $0.05 \%$ of OTU.

The amplicon sequencing taxonomic and statistical analysis were done by the interactive web-interfaced Calypso software, version 8.72 via http:/ / cgenome.net/calypso/ [36] and R software ( $\mathrm{R}$ foundation, Vienna, Austria). Alpha diversity was determined by OTU counts, calculating the Shannon and Simpson's index and assessed by ANOVA. To explore structural differences in the microbial communities among $\mathrm{HpN}, \mathrm{HpP}$, and control groups, a beta diversity analysis was conducted using Permutational Multivariate Analysis of Variance (PERMANOVA) to compare groups using the Bray-Curtis distance metrics. The microbial diversity was visualized by DAPC according to the study group. Biomarker discovery analysis was done by LEfSe, and Wilcoxon tests were 
performed. Identification of co-occurring and mutually exclusive bacteria was performed by the network analysis. Analyses with $p$ value $<0.05$ were considered statistically significant. $p$ value correction included false discovery rate, Bonferroni, or area under curve (AUC) depending on the analysis. Core microbiome analysis was performed based on the presence of taxonomies and its abundance according to comparison groups. The ROC curve analysis was used for selected bacterial biomarkers for $H$. pylori-negative gastritis and species with an AUC in ROC $\geq 0.7$ were included in a multivariate backward logistic regression analysis to select final candidate biomarkers.

\section{Conclusions}

In summary, our findings further support the existence of $\mathrm{HpN}$, and suggest its pathogenic bacteria based on the 16s rRNA amplicon sequencing approach at the species level, which are Streptococcus (OTU ID: 525966), Haemophilus parainfluenza (OTU ID: 4320756) and Treponema (OTU ID: 2707164) as the candidate etiologies. A further long-term clinical follow up and confirmatory experimental studies are required to examine whether these infections are causative pathogenic agents from gastritis to gastric cancer patients.

Supplementary Materials: The following are available online at http:/ /www.mdpi.com/2072-6694/11/4/504/s1. Figure S1: Flow chart for case selection in the three study groups, Figure S2: Overall sequence reads, Figure S3: Rarefaction analysis, Figure S4: Bacterial relative abundance per sample, Figure S5: ANOVA test comparisons for bacterial abundance, Figure S6: Network analysis for comparison groups. Figure S7: Receiver operating curve analysis for selected microbial biomarkers of H. pylori negative gastritis, Table S1: Age and gender status according to the three study groups, Table S2: Selected candidates for microbial biomarkers in H. pylori negative gastritis.

Author Contributions: Conceptualization, B.G., H.B.E.-S., K.O. and Y.Y.; Data curation, B.G., T.M., N.J.A. and T.U.; Formal analysis, B.G., T.M. and N.J.A.; Funding acquisition, H.B.E.-S. and Y.Y.; Investigation, B.G., H.B.E.-S., N.J.A., K.O., T.U. and Y.Y.; Methodology, B.G., H.B.E.-S., T.M. and N.J.A.; Project administration, K.O. and Y.Y.; Resources, B.G., D.A. and Y.Y.; Software, B.G.; Supervision, H.B.E.-S. and K.O.; Validation, B.G.; Visualization, B.G.; Writing—original draft, B.G. and Y.Y.; Writing—review and editing, B.G., H.B.E.-S. and Y.Y.

Funding: This work was supported by Grants-in-Aid for Scientific Research from the Ministry of Education, Culture, Sports, Science and Technology (MEXT) of Japan (15H02657, 16H06279, 16H05191 and 18KK0266) (Y.Y.) and 18K16182 (M.T.), the Special Coordination Funds for Promoting Science and Technology from the MEXT of Japan (Y.Y.), and National Institutes of Health Grants DK62813 (Y.Y.). This material is also based upon work supported by Cancer Prevention \& Research Institute of Texas grant (RP150587) (E.H.B.) and supported in part by the Center for Gastrointestinal Development, Infection and Injury (NIDDK P30 DK 56338) (E.H.B.). B.G. is a doctoral student supported by the Japanese Government (MEXT) Scholarship Program for 2014.

Acknowledgments: The authors thank the Mongolian National University of Medical Sciences researchers for organizing conduct the research among Mongolian population.

Conflicts of Interest: The authors declare no conflict of interest.

\section{References}

1. Conteduca, V.; Sansonno, D.; Lauletta, G.; Russi, S.; Ingravallo, G.; Dammacco, F.H. H. pylori infection and gastric cancer: State of the art. Int. J. Oncol. 2013, 42, 5-18. [CrossRef] [PubMed]

2. Polakovicova, I.; Jerez, S.; Wichmann, I.A.; Sandoval-Bórquez, A.; Carrasco-Véliz, N.; Corvalán, A.H. Role of microRNAs and exosomes in Helicobacter pylori and Epstein-Barr virus associated gastric cancers. Front. Microbiol. 2018, 9, 636. [CrossRef] [PubMed]

3. Noto, J.M.; Peek Jr, R.M. The gastric microbiome, its interaction with Helicobacter pylori, and its potential role in the progression to stomach cancer. PLoS Pathog. 2017, 13, e1006573. [CrossRef] [PubMed]

4. Cisło, M.; Filip, A.A.; Offerhaus, G.J.A.; Ciseł, B.; Rawicz-Pruszyński, K.; Skierucha, M.; Polkowski, W.P. Distinct molecular subtypes of gastric cancer: From Laurén to molecular pathology. Oncotarget 2018, 9, 19427. [CrossRef] [PubMed]

5. Shiota, S.; Thrift, A.P.; Green, L.; Shah, R.; Verstovsek, G.; Rugge, M.; Graham, D.Y.; El-Serag, H.B. Clinical Manifestations of Helicobacter pylori-Negative Gastritis. Clin. Gastroenterol. Hepatol. 2017, 15, 1037-1046. [CrossRef] [PubMed]

6. Ernst, P. The role of inflammation in the pathogenesis of gastric cancer. Aliment. Pharmacol. Ther. 1999, 13, 13-18. [CrossRef] 
7. Rivas-Ortiz, C.I.; Lopez-Vidal, Y.; Arredondo-Hernandez, L.J.R.; Castillo-Rojas, G. Genetic alterations in gastric cancer associated with Helicobacter pylori infection. Front. Med. 2017, 4, 47. [CrossRef]

8. Fu, H.; Ma, Y.; Yang, M.; Zhang, C.; Huang, H.; Xia, Y.; Lu, L.; Jin, W.; Cui, D. Persisting and increasing neutrophil infiltration associates with gastric carcinogenesis and E-cadherin downregulation. Sci. Rep. 2016, 6, 29762. [CrossRef]

9. Warren, J.R. Gastric pathology associated with Helicobacter pylori. Gastroenterol. Clin. N. Am. 2000, 29, 705-751. [CrossRef]

10. Nardone, G.; Compare, D. The human gastric microbiota: Is it time to rethink the pathogenesis of stomach diseases? Un. Eur. Gastroenterol. J. 2015, 3, 255-260. [CrossRef] [PubMed]

11. Parsons, B.N.; Ijaz, U.Z.; D'Amore, R.; Burkitt, M.D.; Eccles, R.; Lenzi, L.; Duckworth, C.A.; Moore, A.R.; Tiszlavicz, L.; Varro, A. Comparison of the human gastric microbiota in hypochlorhydric states arising as a result of Helicobacter pylori-induced atrophic gastritis, autoimmune atrophic gastritis and proton pump inhibitor use. PLoS Pathog. 2017, 13, e1006653. [CrossRef] [PubMed]

12. Bik, E.M.; Eckburg, P.B.; Gill, S.R.; Nelson, K.E.; Purdom, E.A.; Francois, F.; Perez-Perez, G.; Blaser, M.J.; Relman, D.A. Molecular analysis of the bacterial microbiota in the human stomach. Proc. Natl. Acad. Sci. USA 2006, 103, 732-737. [CrossRef] [PubMed]

13. Li, X.-X.; Wong, G.L.-H.; To, K.-F.; Wong, V.W.-S.; Lai, L.H.; Chow, D.K.-L.; Lau, J.Y.-W.; Sung, J.J.-Y.; Ding, C. Bacterial microbiota profiling in gastritis without Helicobacter pylori infection or non-steroidal anti-inflammatory drug use. PLoS ONE 2009, 4, e7985. [CrossRef] [PubMed]

14. Jakobsson, H.E.; Jernberg, C.; Andersson, A.F.; Sjölund-Karlsson, M.; Jansson, J.K.; Engstrand, L. Short-term antibiotic treatment has differing long-term impacts on the human throat and gut microbiome. PLoS ONE 2010, 5, e9836. [CrossRef]

15. Cho, I.; Blaser, M.J. The human microbiome: At the interface of health and disease. Nat. Rev. Genet. 2012, 13, 260. [CrossRef] [PubMed]

16. Nordenstedt, H.; Graham, D.Y.; Kramer, J.R.; Rugge, M.; Verstovsek, G.; Fitzgerald, S.; Alsarraj, A.; Shaib, Y.; Velez, M.E.; Abraham, N. Helicobacter pylori-negative gastritis: Prevalence and risk factors. Am. J. Gastroenterol. 2013, 108, 65. [CrossRef]

17. Sugano, K.; Tack, J.; Kuipers, E.J.; Graham, D.Y.; El-Omar, E.M.; Miura, S.; Haruma, K.; Asaka, M.; Uemura, N.; Malfertheiner, P. Kyoto global consensus report on Helicobacter pylori gastritis. Gut 2015, 64, 1353-1367. [CrossRef]

18. Sohn, S.-H.; Kim, N.; Jo, H.J.; Kim, J.; Park, J.H.; Nam, R.H.; Seok, Y.-J.; Kim, Y.-R.; Lee, D.H. Analysis of gastric body microbiota by pyrosequencing: Possible role of bacteria other than Helicobacter pylori in the gastric carcinogenesis. J. Cancer Prev. 2017, 22, 115. [CrossRef] [PubMed]

19. Snäll, J.; Linnér, A.; Uhlmann, J.; Siemens, N.; Ibold, H.; Janos, M.; Linder, A.; Kreikemeyer, B.; Herwald, H.; Johansson, L. Differential neutrophil responses to bacterial stimuli: Streptococcal strains are potent inducers of heparin-binding protein and resistin-release. Sci. Rep. 2016, 6, 21288. [CrossRef]

20. Liu, J.; Xue, Y.; Zhou, L. Detection of gastritis-associated pathogens by culturing of gastric juice and mucosa. Int. J. Clin. Exp. Pathol. 2018, 11, 2214-2220.

21. Kobus, C.; van den Broek, J.; Richir, M. Acute gastric necrosis caused by a $\beta$-hemolytic streptococcus infection: A case report and review of the literature. Acta Chir. Belg. 2018. [CrossRef]

22. Kosikowska, U.; Biernasiuk, A.; Rybojad, P.; oś, R.; Malm, A. Haemophilus parainfluenzae as a marker of the upper respiratory tract microbiota changes under the influence of preoperative prophylaxis with or without postoperative treatment in patients with lung cancer. BMC Microbiol. 2016, 16, 62. [CrossRef] [PubMed]

23. Middleton, A.; Dowling, R.; Mitchell, J.; Watanabe, S.; Rutman, A.; Pritchard, K.; Tillotson, G.; Hill, S.; Wilson, R. Haemophilus parainfluenzae infection of respiratory mucosa. Respir. Med. 2003, 97, 375-381. [CrossRef]

24. Nwaohiri, N.; Urban, C.; Gluck, J.; Ahluwalia, M.; Wehbeh, W. Tricuspid valve endocarditis caused by Haemophilus parainfluenzae: A case report and review of the literature. Diag. Microbiol. Infect. Dis. 2009, 64, 216-219. [CrossRef] [PubMed]

25. Govind, B.; Veeraraghavan, B.; Anandan, S.; Thomas, N. Haemophilus parainfluenzae: Report of an unusual cause of neonatal sepsis and a literature review. J. Infect. Dev. Ctries 2012, 6, 748-750. [CrossRef] [PubMed] 
26. Aviles-Jimenez, F.; Vazquez-Jimenez, F.; Medrano-Guzman, R.; Mantilla, A.; Torres, J. Stomach microbiota composition varies between patients with non-atrophic gastritis and patients with intestinal type of gastric cancer. Sci. Rep. 2014, 4, 4202. [CrossRef]

27. Dicksved, J.; Lindberg, M.; Rosenquist, M.; Enroth, H.; Jansson, J.K.; Engstrand, L. Molecular characterization of the stomach microbiota in patients with gastric cancer and in controls. J. Med. Microbiol. 2009, 58, 509-516. [CrossRef]

28. Forsythe, S.; Cole, J. Nitrite accumulatin during anaerobic nitrate reduction by binary suspensions of bacteria isolated from the achlorhydric stomach. Microbiology 1987, 133, 1845-1849. [CrossRef]

29. Osman, M.; Hasan, S.; Azher, Q.; Elbedawi, M.; Bachuwa, G. Syphilitic gastritis: A rare presentation of secondary syphilis. Case Rep. 2018, 2018, bcr-2017. [CrossRef]

30. Imhann, F.; Bonder, M.J.; Vila, A.V.; Fu, J.; Mujagic, Z.; Vork, L.; Tigchelaar, E.F.; Jankipersadsing, S.A.; Cenit, M.C.; Harmsen, H.J. Proton pump inhibitors affect the gut microbiome. Gut 2016, 65, 740-748. [CrossRef]

31. Sharaf, R.N.; Shergill, A.K.; Odze, R.D.; Krinsky, M.L.; Fukami, N.; Jain, R.; Appalaneni, V.; Anderson, M.A.; Ben-Menachem, T.; Chandrasekhara, V. Endoscopic mucosal tissue sampling. Gastrointest. Endosc. 2013, 78, 216-224. [CrossRef] [PubMed]

32. Dixon, M.F.; Genta, R.M.; Yardley, J.H.; Correa, P. Classification and grading of gastritis: The updated Sydney system. Am. J. Surg. Pathol. 1996, 20, 1161-1181. [CrossRef] [PubMed]

33. Han, S.; Flamm, R.; Hachem, C.; Kim, H.; Clarridge, J.; Evans, D.; Beyer, J.; Drnec, J.; Graham, D. Transport and storage of Helicobacter pylori from gastric mucosal biopsies and clinical isolates. Eur. J. Clin. Microbiol. Infect. Dis. 1995, 14, 349-352. [CrossRef] [PubMed]

34. Kanada, R.; Uchida, T.; Tsukamoto, Y.; Nguyen, L.T.; Hijiya, N.; Matsuura, K.; Kodama, M.; Okimoto, T.; Murakami, K.; Fujioka, T. Genotyping of the cagA gene of Helicobacter pylori on immunohistochemistry with East Asian CagA-specific antibody. Pathol. Int. 2008, 58, 218-225. [CrossRef]

35. Li, J.; Perez, G.I.P. Is There a Role for the Non-Helicobacter pylori bacteria in the risk of developing gastric cancer? Int. J. Mol. Sci. 2018, 19, 1353. [CrossRef] [PubMed]

36. Zakrzewski, M.; Proietti, C.; Ellis, J.J.; Hasan, S.; Brion, M.-J.; Berger, B.; Krause, L. Calypso: A user-friendly web-server for mining and visualizing microbiome-environment interactions. Bioinformatics 2016, 33, 782-783. [CrossRef] 